\title{
CONF-970503--207
}

\section{Observation of Dark-Current Signals from the S-Band Structures of the SLAC Linac*}

\author{
R. Assmann, F.J. Decker, M. Seidel, R.H. Siemann, D. Whittum \\ Stanford Linear Accelerator Center, Stanford University, \\ Stanford, CA 94309
}

\begin{abstract}
It is well known that the electro-magnetic fields in high-gradient RF structures can cause electron emission from the metallic structure walls. If the emitted electrons are captured and accelerated by the accelerating fields so-called "darkcurrent" is induced. Dark-currents have been measured and studied for various RF-structures. In this paper we present measurements of RF induced signals for the SLC S-band structures. For nominal gradients of $17 \mathrm{MV} / \mathrm{m}$ it is shown that the dark-current can be strong enough to significantly reduce the signalto-noise ratio of the SLC beam wire scanners. We also show results from RF measurements in the dipole band. The measurements are compared to more direct observations of dark-current and it is tried to connect the results to possible effects on the accelerated particle beam.
\end{abstract}

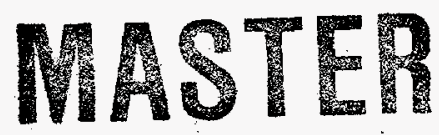

Presented at the 17th IEEE Particle Accelerator Conference (PAC 97): Accelerator Science, Technology and Applications, Vancouver, B.C., Canada, May 12-16, 1997

${ }^{*}$ Work supported by Department of Energy contract DE-AC03-76SF00515. 


\title{
OBSERVATION OF DARK-CURRENT SIGNALS FROM THE S-BAND STRUCTURES OF THE SLAC LINAC ${ }^{1}$
}

\author{
R. Assmann, F.J. Decker, M. Seidel, R.H. Siemann, D. Whittum \\ Stanford Linear Accelerator Center, Stanford University, Stanford, CA 94309
}

\section{Abstract}

It is well known that the electromagnetic fields in highgradient RF structures can cause electron emission from the metallic structure walls. If the emitted electrons are captured and accelerated by the accelerating fields socalled "dark-current" is induced. Dark-currents have been measured and studied for various RF structures. In this paper we present measurements of RF induced signals for the SLC S-band structures. For nominal gradients of $17 \mathrm{MV} / \mathrm{m}$ it is shown that the dark-current can be strong enough to significantly reduce the signal-to-noise ratio of the SLC beam wire scanners. We also show results from RF measurements in the dipole band. The measurements are compared to more direct observations of dark-current and it is tried to connect the results to possible effects on the accelerated particle beam.

\section{INTRODUCTION}

Accelerating, high-gradient RF structures are known to generate significant dark-current [1]. The generation and strength of dark-current has been studied experimentally and theoretically in a number of previous publications $[1,2,3,4]$. Though some quantitative understanding on the strength of dark-currents and its dependence on structure design, cleanliness, gradient, and other parameters has been achieved, it has proved to be hard to quantify the effects on the accelerated beam.

We consider dark-current for the SLC linac. The beam energy ( $>1.19 \mathrm{GeV})$ is high compared to the energy of the dark-current electrons gained within a girder with four or two structures $(<0.22 \mathrm{GeV})$. Due to the large energy disparity, the dark-current is then almost completely lost after the first quadrupole [4]. The dark-current does not propagate down the linac and only local effects are important. These can be twofold:

1. Generation of background for local diagnostic devices.

2. Beam deflections due to possible azimuthal asymmetry in the dark current distribution.

The first nuisance is observed in the SLC linac on a regular basis and results locally in a limitation of maximum accelerating gradient for certain RF structures in the SLC linac. Possible beam deflections due to asymmetric dark-current have not been observed directly so far. They provide, however, a possible mechanism to explain ob- served growth in "beam jitter" along the SLC linac [5]. This mechanism, if strong enough, could generate unacceptably large beam jitter in future linear colliders like the NLC, SBLC or JLC. In this paper we describe attempts to indirectly assess possible effects on the beam by simultaneously measuring the dark-current and RF induced dipole mode signals from the structures.

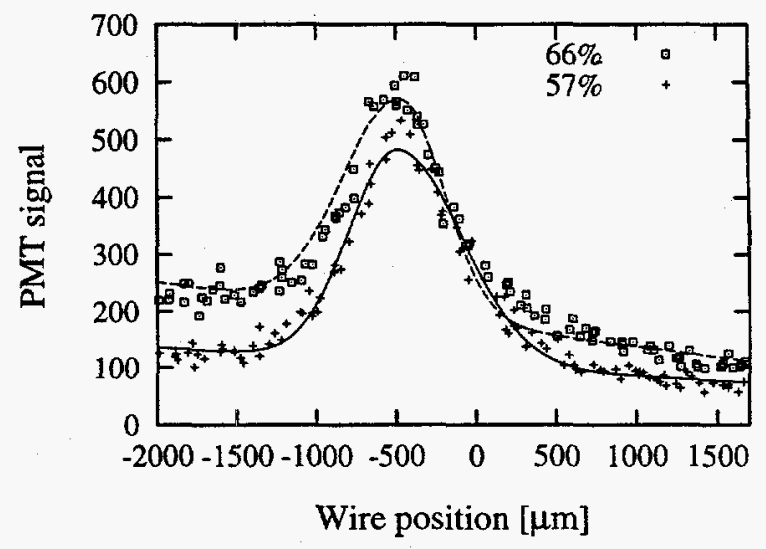

Figure 1 Example of transverse beam profile measurements with two different drive settings of the upstream klystron. The background was almost halved when the klystron drive was reduced from $66 \%$ (squares) to $57 \%$.

\section{SLC WIRE MEASUREMENTS}

$\mathrm{RF}$ induced dark-currents can disturb important beam measurements. Among the most crucial diagnostic devices in SLC are the wire scanners that allow a full reconstruction of the beam emittance at different locations in the linac. A thin wire is moved through the beam and produces scattered electrons. The number of scattered electrons is proportional to the beam intensity at the actual location. A measurement of the number of scattered electrons as a function of wire position then reveals the transverse beam profile.

The scattered electrons are detected with a PhotoMultiplier Tube (PMT) that is located downstream of the moving wire. If dark-current electrons hit the PMT they produce a background to the beam-induced signal. The signal-to-noise ratio is reduced and the measurement is distorted. Figure 1 shows an example where the darkcurrent induced background was reduced by lowering the drive of an upstream klystron.

\footnotetext{
${ }^{1}$ Work supported by the Department of Energy, contract DE-AC03-76SF00515.
} 


\section{DISCLAMIER}

Portions of this document may be illegible in electronic image products. Imsges are produced from the best available original document. 


\section{DISCLAIMER}

This report was prepared as an account of work sponsored by an agency of the United States Government. Neither the United States Government nor any agency thereof, nor any of their employees, make any warranty, express or implied, or assumes any legal liability or responsibility for the accuracy, completeness, or usefulness of any information, apparatus, product, or process disclosed, or represents that its use would not infringe privately owned rights. Reference herein to any specific commercial product, process, or service by trade name, trademark, manufacturer, or otherwise does not necessarily constitute or imply its endorsement, recommendation, or favoring by the United States Government or any agency thereof. The views and opinions of authors expressed herein do not necessarily state or reflect those of the United States Government or any agency thereof. 
Occasionally the dark-current induced background can completely "hide" the beam-induced signal in the SLC linac. As a consequence, the maximum accelerating gradient for certain structures must be limited such that dark-current induced background for the wire measurements is avoided. For the study that follows we used the PMT signals as a qualitative measure of dark-current in the RF structure.

\section{RF INDUCED DIPOLE-MODE SIGNALS}

An asymmetric, not centered current can excite a dipole mode in RF structures. It was shown that beaminduced dipole mode signals correlate well with the centroid position of a particle beam in the RF structure. Here we describe measurements of purely RF induced signals in the dipole-mode frequency range (DM). The setup of dipole-mode measurements for the SLC is described in [6].

In order to study the nature of the observed dipolemode signals, we simultaneously studied RF induced dipole-mode and PMT (compare above) signals. The experimental layout is sketched in Figure 2. We mainly considered C-Band signals at $4.064 \mathrm{GHz}$ and $4.210 \mathrm{GHz}$. The RF induced DM (without beam) produced a signal that was about 60 times smaller (in voltage) than that from a bunch with $3.5 \cdot 10^{10}$ electrons and a $50-100 \mu \mathrm{m}$ offset in the structures.

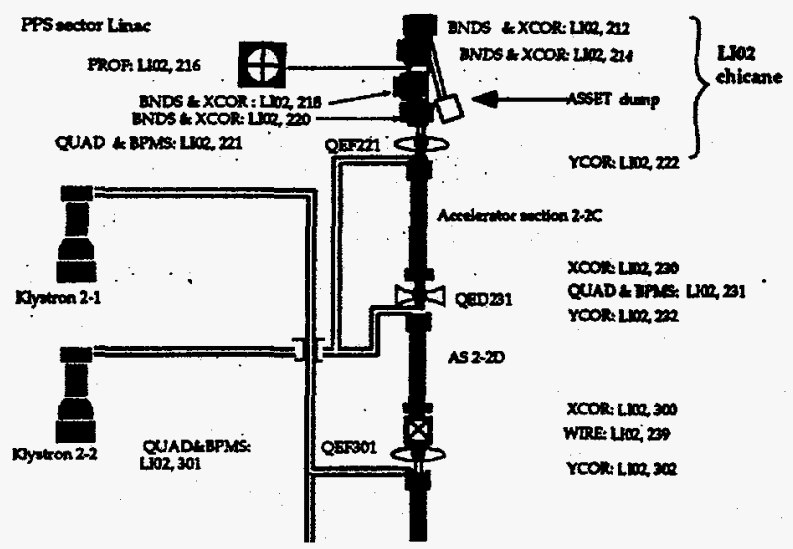

Figure 2 Layout of the experimental setup. The dipolemode signals were measured at the input couplers of Klystron 2-2. The PMT signals were measured at the location of WIRE LI02, 239.

\section{MEASUREMENTS}

RF-induced PMT and C-Band ("DM") signals were measured simultaneously for different klystron parameters. The parameters of interest are:

1. Beam voltage: Voltage of the klystron beam.

2. Klystron delay: Changes delay and width of RF pulse. If small, then the klystron produces no RF power.
3. RF tuning: Tuning of the SLED cavities.

As a first test the klystron beam voltage was changed. Figure 3 shows the measured PMT and C-Band signals for the different settings. The results are summarized in Figure 4. As expected for dark current, the PMT signal shows a strong dependence on the beam voltage. However, the C-Band signal shows only little change.
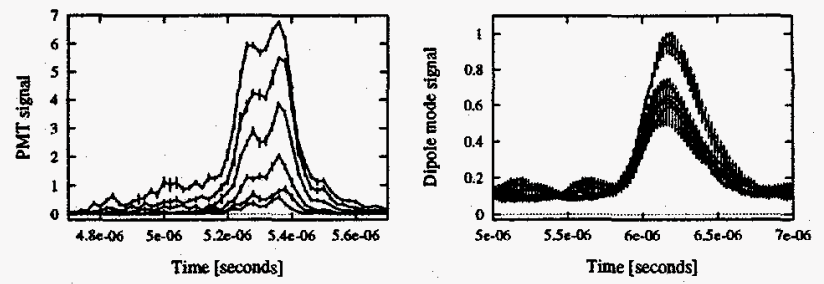

Figure 3 PMT (left) and C-Band (right) signals for different beam voltage settings of klystron 2-2.

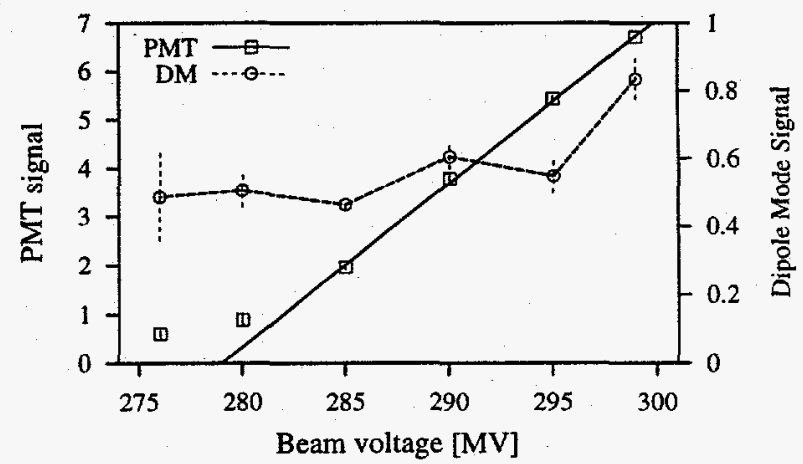

Figure 4 Summary of the results from Figure 3. The PMT signal shows a strong dependence on the klystron beam voltage, while the DM signal does not.

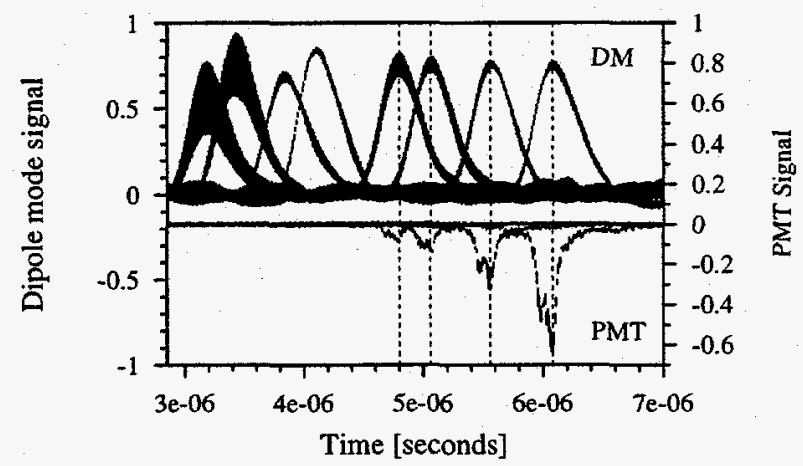

Figure 5 C-Band signals (top) and PMT signals (bottom) for different klystron delays.

The arrival times of both signals follow the klystron delay. This is shown in Figure 5. For small delays the beam voltage drops (measured but not shown here) and the PMT signal vanishes. The C-Band signal becomes unstable (fluctuates between nominal value and zero: large error bars) but does not vanish.

It was also tried to correlate the variations in the PMT and the Beam voltage signals. The correlation for 35 consecutive measurements is shown in Figure 6 . There 
is either no correlation or the correlation is hidden by measurement noise.

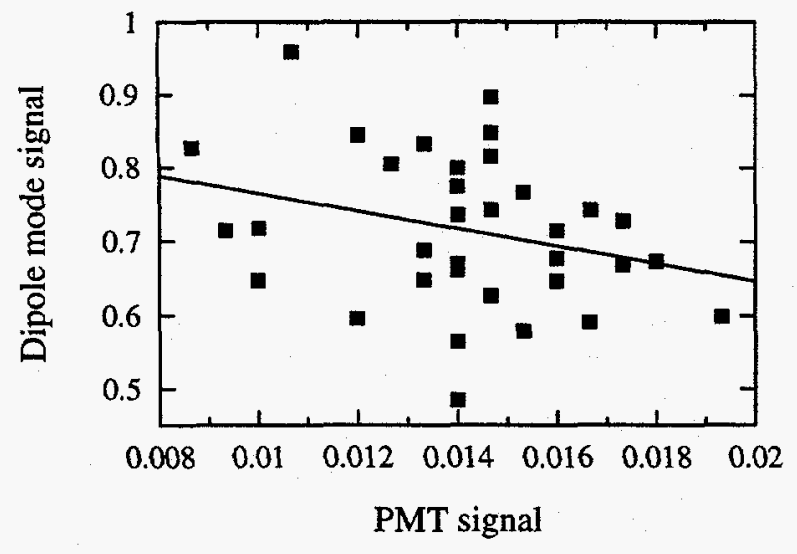

Figure 6 Correlation between C-Band and PMT signal.

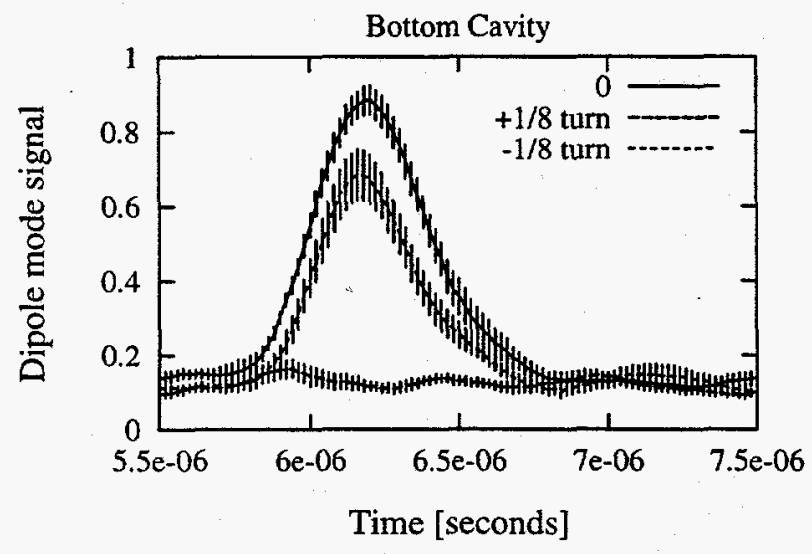

Figure 7 Sensitivity of C-Band signal against tuning of the bottom cavity.

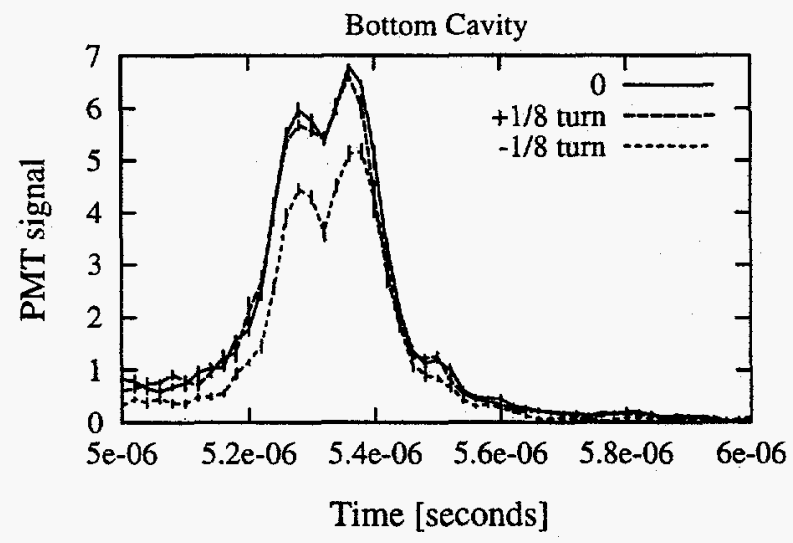

Figure 8 Sensitivity of PMT signal against tuning of the bottom cavity.

Finally we changed the tuning of the SLED cavities. As shown in Figure 7, the C-Band signal was eliminated by a small change in the tuning screw of the bottom cavity. At the same time the PMT dark current signal did not change significantly, indicating that the accelerating RF voltage and the associated dark current did not change.

\section{CONCLUSION}

Dark current in the SLC S-band structures is observed with the photo-multiplier tubes of the wire scanners. We studied the dependence of the PMT signals on several klystron parameters and found the expected qualitative behavior.

We also observed RF-induced C-Band signals. These signal were about 60 times smaller than the dipole mode signal from a bunch with $3.5 \cdot 10^{10}$ electrons and a $50-100 \mu \mathrm{m}$ offset in the structures. We performed measurements at $4.064 \mathrm{GHz}$ and $4.210 \mathrm{GHz}$ for varying klystron parameters. The $\mathrm{C}$-Band signal showed a very different behavior compared to the PMT signal. Its strength did not depend strongly on the beam voltage in the structure or the klystron delay, as did the PMT signal. A minor change in the tuning of a klystron cavity was sufficient to eliminate the C-Band signal. At the same time the klystron beam voltage and the PMT dark current signal remained essentially unchanged. We could adjust the klystron such that we either had a C-Band or a PMT signal. Both signals, generated from the same klystron, apparently are caused by different physical mechanisms.

There is a strong indication that the C-Band signal is generated in the upstairs klystron and not in the RF structure. Because the signal is relatively large, it could affect the beam if it was transported into the downstairs RF structure. We can very roughly estimate that the observed variation in the $\mathrm{C}$-Band signals is equivalent to an rms quadrupole jitter of less than $10 \mathrm{~nm}$. Further studies are needed to get a better quantitative understanding of the strength and importance of klystron-generated RF signals.

\section{ACKNOWLEDGEMENTS}

We thank M. Ross, R. Akre and the SLC RF group.

\section{REFERENCES}

[1] G.A. Loew and J.W. Wang: "Field Emission and RF Breakdown in Copper Linac Structures". Proc. XIV Int. Conf. on High Energy Acc., KEK, Japan, August 22-26, 1989.

[2] H. Matsumoto: “Dark Currents". KEK 96-120.

[3] J. W. Wang et al: "SLAC/CERN High Gradient Tests of an X-Band Accelerating Section". CERN-SL-95$27 \mathrm{RF}$.

[4] N. Akasaka: "Dark Current Simulations in High Gradient Accelerating Structure". KEK 96-65.

[5] C. Adolphsen et al: "Beam Trajectory Jitter in the SLC Linac". PAC95.

[6] M. Seidel, C. Adolphsen, R. Assmann and D. Whittum: "Detection of Beam-Induced Dipole-Mode Signals in the SLC S-Band Structures". These proceedings. 